Running head: INTERINDIVIDUELLE VARIABILITÄT AFFEKTIVER REAKTIONEN

\author{
Zur interindividuellen Variabilität affektiver Reaktionen im Verlauf von Freizeit- und \\ Gesundheitssportprogrammen \\ Gorden Sudeck \\ Institut für Sportwissenschaft, Eberhard Karls Universität Tübingen \\ Achim Conzelmann \\ Institut für Sportwissenschaft, Universität Bern
}

Korrespondenzadresse:

Prof. Dr. Gorden Sudeck, Institut für Sportwissenschaft, Eberhard Karls Universität

Tübingen, Wilhelmstr. 124, 72074 Tübingen

E-Mail: gorden.sudeck@uni-tuebingen.de

Tel.: +4970712976039

Fax: +497071295031 


\title{
Zur interindividuellen Variabilität affektiver Reaktionen im Verlauf von Freizeit- und Gesundheitssportprogrammen
}

\begin{abstract}
Positive Effekte sportlicher Aktivität auf das aktuelle Befinden sind meta-analytisch gut dokumentiert. Eine Generalisierung der Befunde wird dadurch eingeschränkt, dass zunehmend eine hohe interindividuelle Variabilität beobachtet wird und Prozesse während der Aktivität defizitär berücksichtigt wurden. Dieser Beitrag geht den Fragen nach, wie groß das Ausmaß interindividueller Variabilität affektiver Reaktionen im Verlauf von gruppenbasierten Freizeit- und Gesundheitssportaktivitäten ist und welchen Einfluss personale Voraussetzungen (Fitnesszustand) und Faktoren der Auseinandersetzung mit der Aktivität (Beanspruchungserleben, Kompetenzwahrnehmung, positives Gruppenerleben) nehmen. Speziell interessiert, inwieweit affektive Reaktionen in Abhängigkeit vom Zeitpunkt sowie in Abhängigkeit der Intensität von unterschiedlichen Faktoren beeinflusst werden. Dazu wurden 110 Universitätsangestellte $(M=49.5$ Jahre) wiederholt im Rahmen von mehrwöchigen Programmen befragt. An insgesamt sechs Terminen wurden HandheldPC-Befragungen vor, zweimal während und nach der Kursstunde durchgeführt, mit denen das aktuelle Befinden und mögliche Einflussfaktoren erfasst wurden. Die Ergebnisse zeigen, dass die Variabilität affektiver Reaktionen im ersten Abschnitt der Sportprogramme am größten ist, während die Reaktionen am Ende der Kursstunde insgesamt positiver und homogener ausfallen. Im ersten Abschnitt sind personale Voraussetzungen noch wichtige Einflussfaktoren, während die Bedeutung der wahrgenommenen Kompetenz und des positiven Gruppenerlebens im Verlauf der Sportaktivität größer wird. Im Einklang mit der Dual Mode Theory weist die Kompetenzwahrnehmung bei anstrengender Intensität im Vergleich zu moderater Intensität einen größeren Zusammenhang mit dem Befinden auf.
\end{abstract}

Schlüsselwörter: Sportaktivität, Befinden, Dual Mode Theory 
1 Inter-individual variability of acute affective responses in the course of leisure time and

health-oriented exercise programs

\section{Abstract}

4 Meta-analyses document acute positive effects of exercise on affective wellbeing. But, the

5 generalization of these effects is limited due to the increasingly observed high inter-

6 individual variability as well as the lack of consideration for processes during exercise

7 activities. This article focuses on the extent of inter-individual variability of affective

8 responses in the course of group based leisure time and health-oriented exercise programs.

9 Moreover, it deals with the question, which impact personal preconditions (fitness level,

10 affective wellbeing) and subjective experiences of exercise (perceived exertion, perceived

11 competence, and positive group experiences) take. It is of special interest, to what extent

12 affective responses are influenced by varying factors depending on the time within the

13 progress of an exercise session as well as the exercise intensity. Therefore 110 university

14 staff members $(\mathrm{M}=49.5$ years $)$ were asked repeatedly in the context of different exercise

15 programs. On a total of six appointments handheld-PC-surveys were conducted before, twice

16 during and after the session to gather changes in affect and possible influencing factors. The

17 results show that the variability of affective responses is greatest during the first part of a

18 single exercise session whereas in all, the responses are more positive and uniform at the

19 end. Personal preconditions are important influencing factors during the first part of an

20 exercise session. In contrast, the perceived competence and positive group experiences gain

21 in importance over the course of an exercise session. In accordance with the dual mode

22 theory the perceived competence has a greater influence comparing high with moderate

23 intensity.

24 Keywords: Physical Exercise, Affective Response, Dual Mode Theory 
Einleitung

Positive Effekte von sportlichen Aktivitäten auf die psychische Gesundheit sind metaanalytisch gut dokumentiert (z. B. Netz et al., 2005). In zeitlich-dynamischer Hinsicht kann

4 zwischen akuten und habituellen Effekten differenziert werden. Habituelle Effekte beziehen

5 sich z. B. auf die Auswirkungen von mehrwöchigen Sportprogrammen auf die psychische

6 Gesundheit, für die Meta-Analysen in der Regel kleine bis mittlere Effektstärken

7 zusammenfassen (z. B. Netz et al., 2005). Akute Effekte zielen auf unmittelbare

8 Auswirkungen von sportlicher Aktivität auf das aktuelle Befinden, die sowohl für geplante,

9 strukturierte Sportprogramme als auch für körperlich aktive Episoden unter

10 Alltagsbedingungen aufgezeigt werden konnten (vgl. Schlicht, Ebner-Primer \& Kanning,

11 2013). Mittlere Effektstärken in Meta-Analysen zeigen auf, dass hierbei höhere (moderate)

12 Verbesserungen des momentanen Befindens resümiert werden können (Reed \& Ones, 2006).

13 Akute Effekte sportlicher Aktivitäten auf das Befinden sind bereits seit drei Jahrzehnten

14 Gegenstand der Gesundheits- und Sportpsychologie. Im deutschsprachigen Raum hat

15 insbesondere die Arbeitsgruppe um Abele und Brehm (zsfd. Brehm, 1998) in den 1980er-

16 und 1990er Jahren Studien für den Freizeit- und Gesundheitssport veröffentlicht. In ihren

17 Arbeiten stellten sie positive Veränderungen des Befindens durch Sportaktivitäten wie

18 Fitnesskurse, Step Aerobic, Jogging, Fitnesstraining an Geräten und kombinierte

19 Gesundheitssportprogramme fest. Im Vergleich des Befindens vor und nach den

20 Sportaktivitäten zeigten sich Steigerungen in den Kategorien ,Gute Laune', Aktiviertheit und

21 Ruhe, während in den Kategorien Ärger, Deprimiertheit, Erregtheit und Energielosigkeit

22 überwiegend eine Abnahme zu beobachten war. Morgan und $\mathrm{O}^{`} \mathrm{Connor}$ (1988) gingen davon

23 aus, dass sich ca. 80-90\% der Sporttreibenden durch die Aktivität besser fühlen. Ähnlich

24 hielt Brehm (1998) fest, dass die akuten Effekte über verschiedene Studien hinweg bei etwa

$2575 \%$ der Sporttreibenden auftraten. Dem „Feeling Better“-Phänomen wurde daher anfangs

26 ein nahezu universeller Charakter zugeschrieben.

27 Mit Blick auf die Dosierung von Sportaktivitäten wird häufig eine mittlere

28 Belastungsintensität empfohlen (z. B. Brehm, 1998). Auf Basis der Annahme einer

29 umgekehrten U-Funktion zwischen Belastungsdosis und Befinden (z. B. Kirkcaldy \& 
1 Shephard, 1990) soll damit die Wahrscheinlichkeit einer Unter- oder Überforderung

2 reduziert und folglich weniger positive affektive Reaktionen vermieden werden.

\section{Neuere Impulse für die Erforschung affektiver Reaktionen bei Sportaktivitäten}

4 Annahmen über einen nahezu universellen Charakter des „Feeling better“-Phänomens

5 haben sich seit der Jahrtausendwende deutlich relativiert, wobei neuen konzeptionell-

6 methodischen Impulsen nachgegangen wurde (Ekkekakis, Parfitt \& Petruzello, 2011;

7 Schlicht \& Reichertz, 2012). Folgende Gründe sind für diese Weiterentwicklungen zu

8 benennen: Erstens zeigte sich, dass empirische Daten nur unzureichend mit einer

9 umgekehrten U-Funktion in Einklang zu bringen sind, da z. B. auch leichte Aktivitäten mit

10 Verbesserungen des Befindens assoziiert sind (Reed \& Ones, 2006). Zweitens wurde die

11 Notwendigkeit herausgestellt, dass das Befinden während der sportlichen Betätigung zu

12 erfassen sei, um den Verlauf von Befindensveränderungen abbilden zu können (Ekkekakis

13 \& Petruzello, 1999). Diese Forderung kritisiert frühere Studien, in denen mehrheitlich

14 Vorher-Nachher-Messungen realisiert wurden. Am Ende einer Sportaktivität wird aber

15 mitunter die positiv erlebte Vollendung der Aktivität mit erfasst und weniger die affektive

16 Reaktion auf die Aktivität selbst (Backhouse et al., 2007). In der Tat zeigen neuere Studien,

17 dass die Variabilität affektiver Reaktionen während der Aktivität höher einzuschätzen ist als

18 dies nach Beendigung der Aktivität beobachtet werden kann (Van Landuyt et al, 2000; Rose

19 \& Parfitt, 2007). Drittens sollten ausschließliche gruppenbezogene Auswertungen

20 überwunden werden (Ekkekakis \& Petruzello, 1999). Interindividuelle Unterschiede in

21 affektiven Reaktionen sollten nicht mehr nur als zufällige Fehler betrachtet, sondern mit

22 ergänzenden individuumbezogenen Auswertungen (z. B. durch die Darstellung der Anteile

23 von Respondern und Nonrespondern) zu einem systematischen Gegenstand der Analysen

24 gemacht werden (Backhouse et al., 2007; Ekkekakis, et al., 2011).

25 Viertens ist die Frage verstärkt diskutiert worden, wann die Erfassung akuter affektiver

26 Reaktionen auf einer kategorialen oder dimensionalen Befindenskonzeption beruhen sollte.

27 Kategoriale Konzepte, wie sie oben in den operationalisierten Kategorien der

28 Befindlichkeitsskalen (BFS, Abele \& Brehm, 1986) benannt wurden, werden teilweise in

29 theoretischer Hinsicht kritisiert: „The general problem with these approaches is that neither

30 the nature nor the number of distinguable mood states is clear. Moreover, the proposed 
1 specific mood-states are usually highly correlated“" (Wilhelm \& Schoebi, 2007, S. 260). In

2 methodischer Hinsicht sind vorliegende Mehr-Item-Skalen für Befindenskategorien deshalb

3 eher ineffizient, wenn wiederholte Befragungen während und nach sportlicher Aktivität

4 realisiert werden sollen (vgl. ausführlich Ekkekakis, 2008). Dimensionale Ansätze gehen

5 davon aus, dass das aktuelle Befinden über zwei bis drei bipolare Basisdimensionen

6 abgebildet werden kann (z. B. Schimmack und Grob, 2000). Inhaltlich umfassen die

7 Basisdimensionen die affektive Valenz (hedonistische Färbung von angenehm vs.

8 unangenehmen Zuständen), die positive Aktivierung (z. B. energiegeladen vs. energielos)

9 und die Ruhe (auch als negative Aktivierung bezeichnet: z. B. ruhig vs. unruhig; vgl.

10 Wilhelm \& Schoebi, 2007). Für die wiederholte Erfassung situationsspezifischer Einflüsse

11 ergeben sich die Vorteile, dass die Basisdimensionen mit ein bis zwei Items ökonomisch

12 erfasst und relevante affektive Reaktionsinhalte gebündelt beobachtet werden können

13 (Ekkekakis, 2008).

14 Neben diesen konzeptionell-methodischen Aspekten trägt ein fünfter Punkt wesentlich zur

15 Intensivierung der Forschung bei (Ekkekakis et al., 2011): Für affektive Reaktionen bei

16 Sportaktivitäten konnte in prospektiven Studien wiederholt eine Beziehung zum zukünftigen

17 Ausmaß sportlicher Aktivitäten aufgezeigt werden (z. B. Kwan \& Bryan, 2010; Williams,

18 Dunsinger, Jennings \& Marcus, 2012). Die Integration affektiver Reaktionen beim

19 Sporttreiben in die Gesundheitsverhaltensforschung weist deshalb ein großes Potenzial auf

20 (Bryan, Magnan, Nilsson, Marcus, Tompkins \& Hutchinson, 2011).

\section{Einflussfaktoren interindividueller Variabilität affektiver Reaktionen}

22 Affektive Reaktionen weisen eine substanzielle interindividuelle Variabilität auf, die von

23 Verschlechterungen bis hin zu Verbesserungen des Befindens reichen (Van Landuyt et al.,

24 2000). Angesichts dieser Beobachtung stellt sich die Frage nach den Einflussfaktoren auf die

25 interindividuellen Unterschiede: „From a practical standpoint, if the factors that contribute to

26 variability in affective response are identified, this could spur the development of

27 individually tailored interventions, thus optimizing the exercise experience“" (Ekkekakis et

28 al., 2011, S. 657). Einflussfaktoren können personale Voraussetzungen sein oder die

29 unmittelbare Auseinandersetzung mit der Situation des Sporttreibens betreffen. Unter den

30 personalen Voraussetzungen hat sich das Eingangsniveau des Befindens als Moderator von 
1 Befindensveränderungen erwiesen: Je niedriger das Eingangsniveau, desto höher fallen

2 Befindensverbesserungen aus (z. B. Brehm, 1998; Reed \& Ones, 2006, einschließlich von

3 Hinweisen auf methodische Deckeneffekte). Darüber hinaus wurde das bisherige

4 Aktivitätsverhalten bzw. ein guter Fitnesszustand als eine positive Einflussgröße

5 ausgewiesen (Bixby \& Lochbaum, 2007).

Als Merkmal der unmittelbaren Auseinandersetzung mit der Sportaktivität wurde bisher die Intensität der Belastung häufig untersucht (im Überblick Ekkekakis et al., 2011). In ihrer Meta-Analyse über 158 Studien, die den akuten Einfluss von Ausdauertraining auf positiv

9 aktivierten Affekt untersuchten, ermittelten Reed und Ones (2006) unterschiedliche

10 Effektstärken in Abhängigkeit der Intensität: Für leichte $(d=0.45)$ sowie für moderate

11 Aktivitäten $(d=0.46)$ waren mittlere Befindensverbesserungen festzustellen. Bei

12 anstrengenden Aktivitäten ergaben sich im Mittel keine nennenswerten Effekte $(d=0.09)$

13 und bei sehr anstrengenden Aktivitäten waren deutliche Verschlechterungen des Befindens

14 zu beobachten $(d=-0.98)$. Ekkekakis und Kollegen $(2011$, S. 655) folgern aus ihrer

15 systematischen Übersicht, dass eine inverse Beziehung zwischen Intensität und affektiver

16 Reaktion vor allem festzustellen ist, wenn das Befinden während der Aktivität erfasst wird.

17 Als weitere Einflussgröße in der Auseinandersetzung mit der Sportaktivität bestätigte sich

18 wiederholt die Wahrnehmung von Kompetenz. Sowohl in quantitativen (z. B. Welch et al.,

19 2007) als auch qualitativen Studien (Rose \& Parfitt, 2012) wird das Kompetenzerleben bzw.

20 eine hohe Selbstwirksamkeit (d. h. die Überzeugung, die sportiven Anforderungen

21 bewältigen zu können) als Quelle besseren Befindens herausgestellt.

22 Der Bedeutung des sozialen Kontextes auf affektive Reaktionen beim Sporttreiben wurde

23 bisher nur in vereinzelten Studien nachgegangen, da Laborstudien mit Standardisierung von

24 Intensitätsbedingungen dominieren (Ekkekakis et al., 2011). Entsprechend sind theoretische

25 Ansätze mit Bezügen zu sozialen Interaktionen vergleichsweise selten (Lehnert, Sudeck \&

26 Conzelmann, 2012). McAuley und Kollegen (2000) konnten aber in einem Feldexperiment

27 aufzeigen, dass positive Befindensveränderungen in Gruppenangeboten wahrscheinlicher

28 sind, da ein größeres Potenzial für soziale Interaktionen besteht.

29 Ein neuerer Ansatz, die Dual Mode Theory (DMT), bündelt einige der Annahmen und

30 Befunde zur Variabilität affektiver Reaktionen (Ekkekakis, 2003). Die Grundannahmen 
1 basieren auf einem moderierenden Einfluss der Belastungsintensität. Auf Basis

2 evolutionstheoretischer Argumente wird bei Aktivitäten mit moderaten Intensitäten davon

3 ausgegangen, dass die affektiven Reaktionen interindividuell relativ homogen und positiv

4 ausfallen (,pleasure ${ }^{6}$ ). Bei Aktivitäten mit anstrengender Intensität sollen die affektiven

5 Reaktionen interindividuell heterogener sein, da sowohl positive als auch negative

6 Reaktionen auftreten (,pleasure' or ,displeasure'). Erreicht die Aktivität eine sehr anstrengende Intensität fallen die affektiven Reaktionen interindividuell wiederum homogener aus; nun allerdings mit einer negativen affektiven Reaktion (,displeasure‘).

Unterschiede in affektiven Reaktionen werden durch zwei prinzipielle Wirkpfade erklärt

10 die in Abhängigkeit der Intensität zu sehen sind (Ekkekakis, 2003). Bei sehr anstrengenden

11 Aktivitäten (jenseits der ventilatorischen Schwelle) wird ein starker Einfluss interozeptiver

12 Reize angenommen, die z. B. durch Laktatbildung im Muskel oder erschwerter Atmung

13 auftreten. Im anstrengenden Bereich in der Nähe der ventilatorischen Schwelle haben

14 hingegen kognitive Faktoren den größten Einfluss auf die affektive Reaktion. Zu nennen

15 wären hier Unterschiede in der kognitiven Bewertung in der Auseinandersetzung mit der

16 Sportaktivität (z. B. das oben angesprochene Kompetenzerleben), die eine größere

17 interindividuelle Variabilität affektiver Reaktionen hervorbringen. Demgegenüber wird im

18 Bereich moderater Intensitäten, die deutlich unterhalb der ventilatorischen Schwelle liegen,

19 nur ein kleiner bis mittlerer Einfluss kognitiver Faktoren angenommen.

20 Der Schwerpunkt empirischer Bestätigungen zentraler Annahmen der DMT lag auf

21 Differenzierungen zwischen anstrengenden und sehr anstrengenden Aktivitäten mit einer

22 ausdauernden Belastung im Laborkontext (Ekkekakis et al., 2011). Anwendungen der DMT

23 auf die Feldsituation des Freizeit- und Gesundheitssport, die im deutschsprachigen Raum

24 häufig die Besonderheit eines kombinierten Sport- und Bewegungsprogramms (mit

25 Ausdauertraining, Kraft- und Beweglichkeitstraining, spielerische Formen bis hin zu

26 Entspannungsverfahren; z. B. Brehm et al., 2005) im Gruppenkontext aufweist, liegen bisher

27 nicht vor. Für diese Programme ist die Unterscheidung von moderaten und anstrengenden

28 Intensitätsbereichen relevant, da sehr anstrengende Aktivitäten in der Belastungsgestaltung 29 vermieden werden. 
Auf Basis der neueren Forschungsimpulse wird drei Fragenkomplexen nachgegangen:

1) Unterscheidet sich das Ausmaß der interindividuellen Variabilität affektiver

Reaktionen im Verlauf von Freizeit- und Gesundheitssportprogrammen? Dabei wird von

4 einer größeren Variabilität während im Vergleich zum Ende einer Sportaktivität

5 ausgegangen (z. B. van Landuyt et al., 2000).

6 2) Inwieweit stehen interindividuelle Unterschiede in affektiven Reaktionen im

7 Zusammenhang mit personalen Voraussetzungen (Fitnesszustand) und Merkmalen der

8 unmittelbaren Auseinandersetzung mit der Sportaktivität (Belastungsintensität,

9 wahrgenommene Kompetenz und positives Gruppenerleben)? Von Interesse ist, inwieweit

10 diese Zusammenhänge in Abhängigkeit des Zeitpunkts im Sportprogramm variieren. Es

11 werden die Hypothesen geprüft,

12 a) dass der Fitnesszustand die affektiven Reaktion positiv beeinflusst;

13 b) dass ein negativer Einfluss der Belastungsintensität auf die affektive Reaktion besteht,

14 wobei während der Aktivität ein größerer Einfluss als nach Beendigung der Aktivität

15 angenommen wird;

16 c) dass ein positiver Zusammenhang zwischen der wahrgenommenen Kompetenz und den

17 affektiven Reaktionen besteht sowie

18 d) dass ein positiver Zusammenhang zwischen einem positiven Gruppenerleben und den

19 affektiven Reaktionen besteht.

20 3) Unter Anwendung der DMT kann eine moderierende Funktion der

21 Belastungsintensität für den Zusammenhang zwischen kognitiven Faktoren und affektiven

22 Reaktionen angenommen werden. Konkret wird erwartet, dass die wahrgenommene

23 Kompetenz bei anstrengender Belastungsintensität einen größeren Zusammenhang mit den

24 affektiven Reaktionen während der Aktivität hat als bei moderaten Intensitäten.

25 Entsprechend der DMT wird diese Hypothese auf die Befindensdimension der affektiven

26 Valenz bezogen. Explorativ wird diese Frage auf das positive Gruppenerleben erweitert.

\section{Methoden}

28 Die Analysen basieren auf Datenerhebungen im Forschungsprojekt, ,Welcher Sport für 29 wen?“. In einem quasi-experimentellen Design wurde der Wirksamkeit einer motivbasierten

30 Passung von fünf mehrwöchigen Freizeit- und Gesundheitssportprogrammen auf das 
1 aktuelle und habituelle Befinden von Menschen im mittleren Erwachsenenalter

2 nachgegangen (Sudeck \& Conzelmann, 2011). Die Sportprogramme, die in wöchentlichen

3 Einheiten mit einer Dauer von 60 Minuten realisiert wurden, lassen sich inhaltlich wie folgt

4 charakterisieren:

$5-$ Aktiv \& Erholt: Kombination aus Ausdauer- und Krafttraining ohne Geräte; wenn

6 möglich im Freien; kurze Sequenzen bewegungsorientierter Entspannung (ca. 5-10

7 Min.) am Ende der Einheiten

8 - Reload \& Relax: vielfältige Fitnessaktivitäten sowie eine separate Entspannungsperiode

9 am Ende jeder Einheit (15-20 Min.)

$10-$-Zäme Zwäg ' (Zusammen Fit): gesundheitsorientiertes Ausdauer- und Krafttraining

11 sowie Kleine Spiele; interaktives, spielerisches Setting; integrierte Information zu

12 Gesundheitsaspekten

$13-$ SPORT Varia: Kombination aus Ausdauertraining und Sportspielen; wenn möglich im

14 Freien; interaktive Formen

15 - Body \& (E)Motion: Tänzerische und rhythmische Aktivitäten; musikbetonte Gymnastik;

16 kompositorische Elemente

17 Die Untersuchungsmethodik ist weitergehend an anderer Stelle beschrieben (Sudeck,

18 Lehnert \& Conzelmann, 2011; Sudeck \& Conzelmann, 2011), so dass nachfolgend nur die

19 spezifische Methodik zur Bearbeitung der Fragestellungen dargestellt wird.

\section{Untersuchungsgruppe}

21 Die Untersuchungsgruppe setzte sich aus akademischen und nicht-akademischen

22 Angestellten einer Universität in der deutschsprachigen Schweiz zusammen. Die 80 Frauen

23 und 53 Männer waren $\geq 35$ Jahre alt. Das Durchschnittsalter lag bei 49.5 Jahren (SD = 8.3).

24 Die meisten Probanden wiesen als höchsten Bildungsabschluss einen Hochschul- oder

25 Fachhochschulabschluss auf (63\%). Am Beginn der Untersuchung war der Aktivitätsstatus

26 der Teilnehmenden gemischt: Ein Interview anhand eines 7-Day-Recall-Verfahrens (Mäder

27 et al., 2006) ergab, dass $24 \%$ keine Sportaktivitäten betrieben und $6 \%$ weniger als eine

28 Stunde pro Woche sportlich aktiv waren. $21 \%$ der Teilnehmenden betrieb zwischen ein bis

29 zwei Stunden pro Woche Sportaktivitäten, während 23\% zwischen zwei und vier Stunden

30 pro Woche aktiv waren. Weitere $26 \%$ gaben an, mehr als vier Stunden aktiv zu sein. 


\section{Erhebungsverfahren}

2 Eine Anforderung an die Erhebungsmethoden bestand darin, das Geschehen im

3 Sportprogramm so wenig wie möglich zu beeinflussen. Daher mussten die Erhebungen

4 zeitlich stark begrenzt werden. Rheinberg (2004) empfiehlt für Erhebungen während des

5 Tätigkeitsvollzugs eine maximale Dauer von 45 bis 60 Sekunden. Die ökonomische

6 Erfassung von Erlebniskomponenten (etwa durch Single-Items oder Itempaare) ist daher z.

7 B. einer Reliabilitätserhöhung durch Testverlängerung vorzuziehen (Fahrenberg, 2006).

8 Trotz der Kürze der hier eingesetzten Erhebungsverfahren, deren Auswahl, Modifikation

9 oder Neuformulierung durch diese Anforderungen stark geprägt war, zeigt Tab. 1 insgesamt

10 gute Verteilungseigenschaften und zufriedenstellende bis sehr gute interne Konsistenzen auf.

bitte Tabelle 1 hier einfügen

12 Aktuelles Befinden. Das aktuelle Befinden wurde mit einer Kurzskala für die drei

13 affektiven Basisdimensionen erfasst (Wilhelm \& Schoebi, 2007). Die validierte Kurzskala

14 enthält jeweils zwei Adjektivpaare, die als bipolare Ratingfragen zur Erfassung der Valenz

15 (VA: unwohl - wohl, unzufrieden - zufrieden), der positiven Aktivierung (PA: müde -

16 wach, energielos - energiegeladen) und der Ruhe (RU: angespannt - entspannt, unruhig -

17 ruhig) konzipiert wurden. Die Frage „In diesem Moment fühle ich mich...“ war auf einem

18 siebenstufigen Antwortformat zu beantworten, wobei die Endpunkte 0 und 6 mit der

19 Bezeichnung, sehr' gekennzeichnet waren. Für die Analysen wurde jeweils der Mittelwert

20 der beiden Items pro Befindensdimension gebildet, wobei zufriedenstellende bis gute Inter-

21 Item-Korrelationen vorlagen (Tab. 1).

22 Wahrgenommener Fitnesszustand. Der Fitnesszustand wurde als subjektive

23 Einschätzung erfasst. In Anlehnung an die Skala zur Erfassung der wahrgenommenen

24 körperlichen Verfassung (WKV, Kleinert, 2006) wurde die Skala für die Trainiertheit

25 modifiziert und inhaltlich auf die Bereiche der energetisch-determinierten motorischen

26 Fähigkeiten fokussiert. Die Frage „Wie schätzen Sie derzeit Ihre körperliche Verfassung

27 ein?"“wurde mit den Adjektiven ,ausdauernd', ,kräftig' und ,fit' ergänzt, die auf einer

28 sechsstufigen Antwortskala von 0 (,gar nicht') bis 5 (,völlig') zu beantworten waren. Die

29 interne Konsistenz der eingesetzten modifizierten Skala war gut $(\alpha=.89)$. 
1 Wahrgenommene Beanspruchung. Die Belastungsintensität wurde nicht objektiv

2 erfasst, sondern über das subjektive Maß der wahrgenommenen Beanspruchung ermittelt.

3 Hierzu wurde eine Skala in Anlehnung an Buskies und Boeckh-Behrens (2000) eingesetzt,

4 die eine vereinfachte Version der Skala von Borg (1982) darstellt. Während der Aktivität

5 beantworteten die Probanden die Frage „Wie anstrengend haben Sie die Aktivität zuletzt

6 empfunden?“ auf einer siebenstufigen Skala mit wörtlichen Antwortbeschreibungen (1 „sehr

7 leicht", 2 ,leicht", 3 ,eher leicht“, 4 ,mittel“, 5 „,etwas anstrengend“, 6 ,,anstrengend“", 7

8 „sehr anstrengend“). Am Ende der Aktivität sollten die Probanden einschätzen, wie

9 anstrengend sie die Aktivität insgesamt empfunden haben. Ein solches Verfahren, bei dem

10 im Vergleich zur Borg-Skala die Antwortmöglichkeiten reduziert werden, wird von Buskies

11 und Boeckh-Behrens empfohlen, wenn bei weniger erfahrenen Aktiven keine vorbereitenden

12 Übungen zur Beantwortung einer differenzierteren Borg-Skala möglich sind.

13 Wahrgenommene Kompetenz. Ein Einzelitem wurde eingesetzt, um die

14 wahrgenommene Kompetenz bei der sportlichen Betätigung zu erfassen. Dieses Item wurde

15 in Anlehnung an die ,Challenge-skill balance' der Flow State Scale (Jackson \& Marsh,

16 1996) formuliert. Auf die Eingangsfrage "Wenn Sie an die heutige Kursstunde denken:

17 Welche Erlebnisse hatten Sie bzw. Erfahrungen haben Sie gemacht?" musste das Statement

18 "Gefühl, kompetent genug zu sein, um die Anforderungen in der Kursstunde zu erfüllen" auf

19 einer sechsstufigen Skala (,trifft nicht zu“(0) bis „trifft völlig zu“(5)) beantwortet werden.

20 Positives Gruppenerleben. Die Wahrnehmung eines positiven Gruppenerlebens wurde

21 mit zwei Items erfasst, die für diese Untersuchung neu formuliert wurden. Auf die oben

22 benannte Eingangsfrage waren die Aussage ,angenehme Gruppenatmosphäre ‘ und

23 ,geselliges Miteinander‘ auf der sechsstufigen Ratingskala zu beantworten. Für die Analysen

24 wurde der Mittelwert der Items verwendet, die eine zufriedenstellende Inter-Item-

25 Korrelation aufwiesen.

26 Untersuchungsdurchführung

27 Die Sportprogramme wurden in zwei Semestern im Rahmen des universitären

28 Hochschulsports über 13 bzw. 14 Wochen hinweg wöchentlich angeboten. In zwei

29 Rekrutierungsphasen wurden Angestellte per Post und per E-Mail angeschrieben und über

30 das Projekt informiert. Nachdem 228 Interessierte ein umfangreiches Assessment 
1 individueller motivationaler, körperlicher und aktivitätsbezogener Voraussetzungen

2 durchgeführt hatten, wurden fünf Sportprogramme mit einer motivbasierten

3 Maßschneiderung auf verschiedene Segmente der Zielgruppe entwickelt und durchgeführt

4 (Sudeck et al., 2011; Sudeck \& Conzelmann, 2011). 133 Angestellte wählten auf Basis einer

5 Kurzbeschreibung eines der fünf Sportprogramme aus. Nach Information über die Ziele und

6 Ablauf des Forschungsprojekts meldeten sie sich auf freiwilliger Basis schriftlich für das

selbstgewählte Sportprogramm an und erklärten sich mit der Teilnahme am

8 Forschungsprojekt einverstanden. In Semester 1 starteten 103 Angestellte ihre

9 Kursteilnahme (vgl. Abb. 1). In Semester 2 begannen 30 Angestellte, während die Anderen

10 ihre Teilnahme fortsetzten $(n=66)$ oder nicht fortsetzten $(n=37)$.

bitte Abbildung 1 hier einfügen

12 Datenerhebungen wurden in Semester 1 und 2 jeweils in drei Kursstunden per Handheld-

13 PCs durchgeführt. Diese Befragungen wurden mit der Software IzyBuilder 2.0 und dem

14 Handheld-PC HP iPAQ114 realisiert. In Semester 1 wurden die Datenerhebungen in den

15 Kurswochen 8, 10 und 12 durchgeführt, während sie Semester 2 in den Kurswochen 3, 7 und

1611 durchgeführt wurden. Abbildung 1 gibt die tatsächliche Anzahl der Befragungen pro

17 Person wieder, die von 0 bis 6 (Teilnahme in beiden Semestern) bzw. von 0 bis 3 (Teilnahme

18 in einem Semester) reichen konnte. Insgesamt nahmen 110 der 133 Kursteilnehmenden

19 mindestens einmal an einer Handheld-Befragung teil. Diese Subgruppe unterschied sich zu

20 Untersuchungsbeginn in der Geschlechterverteilung von der Subgruppe der Personen, die

21 nicht erreicht werden konnte $\left(\chi^{2}(1)=12.6\right.$; $\left.p<\alpha_{\text {krit }}=.20\right)$, während es keine Unterschiede

22 hinsichtlich Alter, höchstem Bildungsabschluss und Aktivitätsverhalten gab $\left(\mathrm{p}>\alpha_{\text {krit }}=.20\right) .{ }^{1}$

23 In den Kursstunden mit Handheld-Befragungen wurden vier Erhebungen durchgeführt

24 (Abb. 1): Eine Befragung vor Beginn der Kursstunde (A), zwei während der Kursstunde

25 (W1, W2) und eine Befragung am Ende der Kursstunde (E). Der Zeitpunkt der Während-

26 Befragungen war nicht minutengenau fixiert, sondern richtete sich nach dem Aufbau der

27 Kursstunde, so dass in der Regel nach Abschluss eines Stundenteils die Befragung in den

\footnotetext{
${ }^{1}$ In der Konsequenz wurden alle Berechnungen für die drei Fragestellungen mit und ohne die Kovariate Geschlecht berechnet. Die statistischen Entscheidungen blieben jedoch vom dem Einbezug der Kovariate unbeeinflusst, so dass im Ergebnisteil die Analysen ohne Kovariate Geschlecht dargestellt werden.
} 
anvisierten Zeitfenstern zwischengeschaltet wurde. Die Tab. 1 gibt einen Überblick, welche

2 der Erhebungsverfahren in A, W1, W2 und E eingesetzt wurden.

\section{Datenanalyse}

4 Zunächst wurde per Zufall ein Erhebungstermin pro Proband ausgewählt. Dieses

5 Vorgehen wurde gewählt, da zum einen die zur Verfügung stehenden Daten pro Person

6 aufgrund der unterschiedlichen Anwesenheiten bei den Erhebungsterminen stark variierten. ${ }^{2}$

7 Zum anderen sollte auf eine Aggregation der vorhandenen Daten verzichtet werden, um das

8 konkrete Erleben in einer spezifischen Kursstunde abbilden zu können. Eine zufällige

9 Auswahl wurde getroffen, um einen möglichen Einfluss des Zeitpunkts innerhalb der

10 mehrwöchigen Sportprogramme zu minimieren. Auf diese Weise ergab sich eine relative

11 Gleichverteilung über die sechs Termine mit Handheld-Befragung ( $15<n<20$ pro Termin).

12 Innerhalb dieser ausgewählten Kursstunden konnte ein relativ hoher Anteil vollständiger

13 Datensätze realisiert werden (vgl. Abb. 1). Lediglich bei der W2-Befragung reduzierte sich

14 die Anzahl der befragten Probanden auf 97. Der Grund lag darin, dass zu Beginn der

15 Datenerhebungen die zweite Befragung während der Sportaktivitäten in zwei

16 Sportprogrammen nicht realisiert wurde, da die Kursleitenden entgegen der Instruktion eine

17 weitere Unterbrechung während der Kursstunde vermieden.

18 Für die erste Fragestellung wurden die affektiven Reaktionen anhand der Rohwerte sowie

19 einfacher Differenzwerte des aktuellen Befindens im Vergleich zum Eingangswert deskriptiv

20 betrachtet. Unterschiede in den Varianzen des aktuellen Befindens bei den Erhebungen W1,

21 W2 und E wurden anhand von F-Tests statistisch geprüft $\left(\alpha_{\text {krit }}=.05\right)$. Hierbei wurde eine

22 statistische Kontrolle für den Eingangswert und den Einfluss der fünf Sportprogramme vorgenommen. ${ }^{3}$

24 Der zweiten Fragestellung wurde anhand blockweiser linearer Regressionsanalysen nachgegangen. Die drei affektiven Basisdimensionen wurden separat als abhängige Variable berücksichtigt. Das blockweise Vorgehen bezog in Schritt 1 die fünf Sportprogramme als

27 Kovariate anhand von vier Dummy-Variablen ein. Schritt 2 berücksichtigte das Eingangs-

\footnotetext{
${ }^{2}$ Auf einen systematischen Einbezug der intraindividuellen Varianz über die verschiedenen Kurseinheiten hinweg (z. B. durch die Verwendung von Multi-Level-Analysen) wurde aufgrund dieser Einschränkung in den vorhandenen Informationen pro Proband verzichtet.

${ }^{3}$ Für die statistische Kontrolle wurden jeweils für die Befindensdaten zu W1, W2 und E autoregressive Modelle mit dem Eingangswert und der Kovariate Kursart (anhand von vier Dummy-Variablen) berechnet. Die vorhergesagten unstandardisierten Werte wurden nachfolgend in den Auswertungen herangezogen.
} 
1 Befinden als weitere Kovariate. Schritt 3 bezog sich auf den zusätzlichen Einfluss des

2 wahrgenommenen Fitnesszustands als personale Voraussetzung und in Schritt 4 wurden die

3 subjektiven Erfahrungen mit der Sportaktivität (wahrgenommene Beanspruchung,

4 wahrgenommene Kompetenz, positives Gruppenerleben) ergänzt. Zur Beantwortung der

$5 \quad$ Fragestellung wurden Veränderungen des Determinationskoeffizienten durch den Einschluss

6 der zusätzlichen Prädiktoren herangezogen $\left(\Delta \mathrm{R}^{2}\right)$ sowie die Regressionsgewichte der einzelnen Prädiktoren betrachtet $(\beta)$. Das kritische Signifikanzniveau wurde konventionsgemäß auf $\alpha_{\text {krit }}<.05$ festgelegt. Die Regressionsanalysen wurden getrennt für die Erhebungen W1, W2 und E berechnet.

Für die dritte Fragestellung wurden Kategorien für die Beanspruchung gebildet, wobei

11 Antwortbeschreibungen in Anlehnung an Kategorien der Borg-Skala (ACSM, 2011)

12 eingeordnet wurden. Die Werte 3 (eher leicht) und 4 (mittel) wurden als moderate Aktivität

13 eingeordnet und die Werte 5 (eher anstrengend) und 6 (anstrengend) als anstrengende

14 Aktivität klassifiziert. ${ }^{4}$ Die Auswertung erfolgte analog der Vorgehensweise für die zweite

15 Fragestellung. Diese blockweisen Regressionsanalysen wurden separat für moderate oder anstrengende Aktivitäten berechnet. Eine statistische Unterschiedsprüfung wurde bei einseitiger Testung anhand der 90\%-Konfidenzintervalle der unstandardisierten

Regressionsgewichte bei moderater im Vergleich zu anstrengender Aktivität vorgenommen.

Die individuumsbezogenen Auswertungen anhand der Richtung der einfachen

21 Differenzwerte geben erste Antworten für die Fragestellung 1. Bei der W1-Befragung sind die geringsten Anteile der Personen festzustellen, die bereits ein gesteigertes Befinden angaben (VA: 44\%, RU: 53\%; PA: 58\%). Bei W2 liegt eine Steigerung des Befindens bei $66 \%$ (VA, RU) bis 71\% (PA) der Teilnehmenden vor. Am Ende berichteten zwischen 70\% (VA), 73\% (PA) und 75\% (RU) der Teilnehmenden ein gesteigertes Befinden. Hierbei ist allerdings zu berücksichtigen, dass teilweise Personen bereits vor der Kursstunde den

27 Höchstwert 6 angaben. Dies trat bei VA am häufigsten auf $(n=11)$ und war bei RU $(n=4)$ und PA $(n=1)$ seltener. In diesem Fall können unveränderte Befindenswerte als positive

\footnotetext{
${ }^{4}$ Beanspruchungen mit dem Wert 7 (sehr anstrengend) wurden nicht berücksichtigt, da die DMT für diese Beanspruchungskategorie andere Aussagen über Einflussfaktoren macht. Erwartungsgemäß war dies nur selten der Fall (W1: $n=0$; W2: $n=2$ ). Beanspruchung mit dem Wert 1 (sehr leicht) oder 2 (leicht) wurden ebenso aus theoretischen Gründen nicht berücksichtigt. Dies traf für $n=23$ (W1) bzw. $n=10$ Probanden (W2) zu.
} 
1 Reaktion betrachtet werden. Exemplarisch für VA trat dieser Fall bei 6 (W1), 3 (W2) bzw. 8

2 (E) Personen auf, so dass der Anteil mit positiver Reaktion mit 50\% (W1), 69\% (W2) bzw.

$378 \%$ (E) der Teilnehmenden höher zu beziffern ist. ${ }^{5}$ Der Anteil der Personen mit einer

4 Abnahme des Befindens reduziert sich im Verlauf der Stunde. Bei W1 ist ein vermindertes

5 Befinden bei 17\% (PA), 19\% (VA) bzw. 27\% (RU) der Teilnehmenden festzustellen. Bei

6 W2 liegt dieser Anteil zwischen 18\% (RU) und 20\% (VA, PA) und am Ende ist eine

7 Abnahme bei 13\% (VA, PA) bzw. 14\% (RU) zu verzeichnen.

In Abb. 2 sind die Befindenswerte im Verlauf der Sportprogramme dargestellt, die für den

9 Eingangswert und die Kursart kontrolliert wurden. Zunächst lassen diese gruppenbezogenen

10 Auswertungen wieder erkennen, dass für alle drei Befindensdimensionen im Verlauf der

11 Sportprogramme im Mittel sukzessive Steigerungen vorliegen. Die Variabilität der

12 affektiven Valenz (VA) und der Ruhe (RU) nimmt sukzessive ab (VA: $\mathrm{SD}_{\mathrm{W} 1}=0.55 ; \mathrm{SD}_{\mathrm{W} 2}=$

$\left.130.26 ; \mathrm{SD}_{\mathrm{E}}=0.19 ; \mathrm{RU}: \mathrm{SD}_{\mathrm{W} 1}=0.55 ; \mathrm{SD}_{\mathrm{W} 2}=0.32 ; \mathrm{SD}_{\mathrm{E}}=0.25\right)$. Erwartungskonform zeigen

14 sich statistisch signifikante Varianzunterschiede im Vergleich der Befindenswerte während

15 und am Ende der Sportaktivität (je $F \geq 1.52>F_{\text {krit, 100,100 }}=1.39$ ) und darüber hinaus

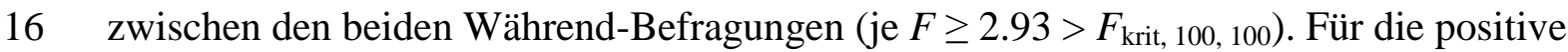

17 Aktivierung (PA) zeigen sich ebenso Unterschiede zwischen der Befragung am Stundenende und der W1- bzw. W2-Befragung $\left(\mathrm{SD}_{\mathrm{W} 1}=0.36 ; \mathrm{SD}_{\mathrm{W} 2}=0.39 ; \mathrm{SD}_{\mathrm{E}}=0.21\right.$; je $F \geq 3.00>$

$19 F_{\text {krit, 100, 100). }}$

bitte Abbildung 2 hier einfügen

Für die Einflussfaktoren und Korrelate affektiver Reaktionen im Verlauf der

Sportprogramme (Fragestellung 2) zeigen die Regressionsanalysen zwei prinzipielle

23 Ergebnisrichtungen (Tab. 2): Erstens nehmen die Einflüsse des Eingangs-Befindens ( $\Delta \mathrm{R}^{2}$,

24 Step 2) und des Fitnesszustands ( $\Delta \mathrm{R}^{2}$, Step 3) im Verlauf der Sportaktivität ab. Das

25 Eingangs-Befinden ist insbesondere bei der W1-Befragung der stärkste Prädiktor für VA und

26 RU. Der Einfluss des Fitnesszustands zeigt sich für PA in allen drei Messungen W1, W2 und

27 E (mit abnehmender Varianzaufklärung). Für die Valenz zeigt sich nur bei W1 ein Einfluss

28 des Fitnesszustands, wohingegen das Erleben von Ruhe unabhängig vom Fitnesszustand ist.

\footnotetext{
${ }^{5}$ Bei RU trat der Fall nur bei einer Person am Ende der Kursstunde auf. Die eine Person mit dem Höchstwert für PA blieb im Verlauf der Kursstunde auf diesem Niveau.
} 
1 Zweitens nehmen die Zusammenhänge zwischen subjektiven Erfahrungen mit der

2 Sportaktivität und dem Befinden mit zunehmender Dauer der Aktivität zu ( $\Delta \mathrm{R}^{2}$, Step 4). Für

3 die relative Bedeutung der einzelnen Merkmale zeigt sich für alle drei

4 Befindensdimensionen, dass das Befinden am Ende der Kursstunde sowohl mit dem

5 Kompetenzerleben $(.24<\beta<.29)$ als auch mit dem positiven Gruppenerleben $(.28<\beta<.45)$

6 signifikant zusammenhängt. Für die wahrgenommene Beanspruchung sind am Ende der

7 Kursstunde keine signifikanten Regressionsgewichte zu verzeichnen.

8 Die wahrgenommene Beanspruchung weist jedoch während der Aktivität negative

9 Zusammenhänge mit den drei Befindensdimensionen auf. Allerdings erreichen nur drei der

10 sechs geprüften Regressionsgewichte das statistische Signifikanzniveau. Die

11 wahrgenommene Kompetenz zeigt ab der W2-Befragung konstante Zusammenhänge mit

12 den drei Befindensdimensionen $(.24<\beta<.31)$. Beim Zeitpunkt W1 liegt für dieses Merkmal

13 nur für das Kriterium affektive Valenz ein signifikanter $\beta$-Koeffizient vor. Das positive

14 Gruppenerleben weist signifikant positive Regressionsgewichte für VA und PA während der

15 Aktivität auf. Das Gruppenerleben zeigt dabei für VA die größten Zusammenhänge $(.20<\beta$

$16<.36)$; sie sind aber auch bei PA zu jedem Zeitpunkt statistisch signifikant $(.17<\beta<.18)$.

17 Für das Erleben von Ruhe ist hingegen kein signifikantes Regressionsgewicht für das

18 Gruppenerleben festzustellen.

bitte Tabelle 2 hier einfügen

20 Entsprechend der Erwartungen zeigen die Regressionsanalysen für die dritte

21 Fragestellung, dass die wahrgenommene Kompetenz einen stärkeren Zusammenhang mit

22 der affektiven Valenz aufweist, wenn die Aktivität als anstrengend erlebt wird (Tab. 3). Die

23 Regressionsgewichte sind bei anstrengender Aktivität jeweils statistisch signifikant (W1: $\beta=$

$24.54 ; \mathrm{W} 2: \beta=.45$ ), während dies bei moderater Aktivität nicht der Fall ist (W1: $\beta=.10$; W2:

$25 \beta=.14)$. Den Konfidenzintervallen der unstandardisierten Regressionskoeffizienten kann

26 entnommen werden, dass sich die Zusammenhänge zwischen Kompetenzerleben und

27 affektiver Valenz sowohl bei W1 (moderat: B = 0.12; CI 90\%: [-0.05-0.29]; anstrengend:

$28 \mathrm{~B}=0.41$; CI 90\%: [-0.13 - 0.69]) als auch bei W2 (moderat: $\mathrm{B}=0.12$; CI 90\%: [-0.09 -

29 0.33]; anstrengend: B = 0.56; CI 90\%: [0.25 - 0.87]) statistisch signifikant in Abhängigkeit

30 der wahrgenommenen Beanspruchung unterscheiden. 


\section{bitte Tabelle 3 hier einfügen}

Demgegenüber erreicht das positive Gruppenerleben nur bei moderater Aktivität ein signifikantes Regressionsgewicht. Dieses Ergebnismuster zeigt sich erst in der zweiten Während-Befragung $\left(\beta_{\text {moderat }}=.50 ; \beta_{\text {anstrengend }}=.15\right)$. Bei der ersten Während-Befragung weisen weder das positive Gruppenerleben noch die wahrgenommene Kompetenz bei moderater Aktivität signifikante Regressionsgewichte auf. Bei moderater Aktivität ist es vielmehr der Eingangswert des Befindens, der in der W1-Befragung den stärksten Zusammenhang mit der affektiven Valenz hat.

\section{Diskussion}

Die Studienergebnisse verdeutlichen, dass die interindividuelle Variabilität affektiver Reaktionen in Freizeit- und Gesundheitssportprogrammen vom Zeitpunkt der Erfassung des Befindens abhängig sind. Die berücksichtigten neueren konzeptionell-methodischen Impulse zur Erforschung affektiver Reaktionen während und nach Sportaktivitäten können demnach für den Anwendungsbereich von gruppenbasierten Programmen im Freizeit- und Gesundheitssport relevante Erkenntnisse für das Ausmaß und die Bedingungen interindividueller Unterschiede in affektiven Reaktionen liefern.

\section{Ausmaß interindividueller Variabilität (Fragestellung 1)}

Die Auswertungen für die Fragestellung 1 bestätigen frühere Befunde, dass die Variabilität des affektiven Befindens während der Aktivität größer ist als nach Beendigung der Aktivität (van Landuyt et al., 2000; Rose \& Parfitt, 2007). Nach 10-15 Min. während der Aktivität war die affektive Valenz bei jedem dritten Teilnehmenden noch unverändert und bei jedem fünften Teilnehmenden hatte eine Abnahme stattgefunden. Im Verlauf der Sportprogramme wurden die affektiven Reaktionen dann aber sukzessive (Valenz und Ruhe) bzw. am Ende der Aktivität (positive Aktivierung) homogener und positiver. In der Konsequenz lassen sich erst nach Beendigung der 60-minütigen Kursstunden jene Anteile von Personen mit positiven affektiven Reaktionen feststellen, die auf Basis von VorherNachher-Vergleichen im Freizeit- und Gesundheitssport bei ca. 75\% berichtet wurden (z. B. Brehm, 1998). Eine höhere Variabilität während im Vergleich zur Vollendung einer Sportaktivität kann somit auch für Programme beobachtet werden, die nicht als einförmige Ausdauerbelastungen, sondern als Kombination von Aktivitätstypen konzipiert sind. 
1 Befindensabfragen am Ende von Programmstunden bilden daher nicht mehr allein die

2 affektive Reaktion auf die sportliche Aktivität selbst ab, sondern sie sind von der

3 Beendigung dieser Aktivität mitbeeinflusst (Backhouse et al., 2007). So können bereits

4 kognitive Bewertungen bedeutsam sein, die auf das Vollbringen einer - ggf. affektiv negativ

5 besetzten - anstrengenden Sportaktivität ausgerichtet sind, die z. B. mit einem positiven

6 gesundheitlichen Nutzen verbunden wird (z. B. „Ich habe etwas Gutes für meine Fitness und 7 Gesundheit getan“).

8 Einschränkend müssen zwei Aspekte betont werden: Erstens konnten die Auswertungen

9 nicht auf den Einfluss des jeweiligen Aktivitätstyps eingehen. In dieser Studie wurden bei

10 den Befragungszeitpunkten unterschiedliche Aktivitäten in den verschiedenen Kursstunden

11 und -programmen durchgeführt. Eine Konfundierung von Aktivitätstyp und Zeitpunkt in der

12 Kursstunde kann nicht ausgeschlossen werden. Für die Interpretation der ersten

13 Fragestellung kann dies z. B. bedeuten, dass neben dem Effekt der Vollendung einer

14 Sportaktivität der Abschlussteil bzw. das Cool-Down in den Freizeit- und

15 Gesundheitssportprogrammen für eine interindividuelle Homogenisierung und Positivierung

16 des Befindens mit verantwortlich ist. Zweitens bestand die Untersuchungsgruppe

17 mehrheitlich aus bereits sportlich aktiveren Personen. Ein größerer Einbezug von sportlich inaktiveren Personen oder Personen mit gesundheitlichen Risikofaktoren könnte die aufzufindende Variabilität affektiver Reaktionen beeinflussen, wobei eher eine noch größere interindividuelle Variabilität zu vermuten wäre (z. B. Ekkekakis, Lind \& Vazou, 2010).

Eine größere Variabilität affektiver Reaktionen während sportlicher Aktivität kann ihre Bedeutung vor allem im Hinblick auf die Regelmäßigkeit sportlicher Aktivitäten besitzen, wenn sich z. B. negative affektive Assoziationen auf die sportliche Betätigung selbst

24 beziehen (trotz der Erwartung, dass das Befinden am Ende einer sportlichen Aktivität mit hoher Wahrscheinlichkeit gesteigert sein dürfte). Vor diesem Hintergrund wurden affektive

26 Reaktionen erstmalig in Empfehlungen des American College of Sports Medicine (ACSM,

27 2011) für ein individualisiertes Training aufgegriffen und als sekundärer Parameter der

28 Belastungssteuerung während sportlicher Aktivität eingeführt (Ekkkakis et al., 2011). 
1 Für die zweite Fragestellung ergaben sich differenzielle Befunde in Abhängigkeit des

2 Zeitpunkts der Befragung sowie in Abhängigkeit der Befindensdimension. Der positive

3 Einfluss des Fitnesszustands bestätigte sich für die positive Aktivierung sowohl während als

4 auch am Ende der Aktivität. Demgegenüber waren die Valenz nur im ersten Abschnitt der

5 Kursstunde und die Ruhe gar nicht vom Fitnesszustand beeinflusst. Dieses Befundmuster ist

6 plausibel, insofern die positive Aktivierung die am stärksten energetisch determinierte

7 Befindensdimension darstellt.

8 Der Einfluss der Belastungsintensität auf die affektiven Reaktionen zeigte sich

9 erwartungskonform in negativer Richtung sowie stärker während als am Ende der Aktivität.

10 Während der Aktivität wiesen die Hälfte, nicht jedoch alle geprüften Regressionsgewichte,

11 eine statistische Signifikanz auf. Es kann deshalb von einer partiellen Bestätigung der

12 Hypothese gesprochen werden.

13 Der Zusammenhang zwischen der wahrgenommenen Kompetenz bzw. eines positiven

14 Gruppenerlebens und den affektiven Reaktionen zeigte sich wie erwartet in positiver

15 Richtung. Am Ende der Kursstunden bestand dieses Ergebnismuster für alle drei

16 Befindensdimensionen. Während der Aktivität waren differenzielle Zusammenhänge in

17 Abhängigkeit der Befindensdimension zu sehen. Die Kompetenzwahrnehmung zeigte im

18 früheren Stundenabschnitt erst Zusammenhänge mit der affektiven Valenz, um dann im

19 späteren Verlauf mit allen Befindensdimensionen einen positiven Zusammenhang

20 aufzuweisen. Insgesamt wird dadurch die bestehende Befundlage zur wesentlichen

21 Bedeutung des Kompetenzerlebens für affektive Reaktionen bekräftigt (z. B. Rose \& Parfitt,

22 2012; Welch et al., 2007). Ein positives Gruppenerleben stand während der Aktivität im

23 Zusammenhang mit der affektiven Valenz und der positiven Aktivierung. Die

24 Anregungsbedingung sozialer Interaktionen hing folglich nicht substanziell mit dem

25 Ruheerleben zusammen. Dieses Ergebnismuster erscheint prinzipiell plausibel, insofern

26 soziale Aktivitäten unmittelbar weniger ruheförderlich sind, als vielmehr aktivierenden

27 Charakter aufweisen.

28 In der Gesamtbetrachtung der zweiten Fragestellung haben sich die erwarteten

29 Einflussrichtungen der untersuchten Faktoren bestätigt. Dabei ist zu betonen, dass das

30 Kompetenzerleben und das positive Gruppenerleben mehrheitlich unabhängig voneinander 
1 Zusammenhänge mit affektiven Reaktionen aufweisen, die bei simultaner Betrachtung

2 multipler Prädiktoren bestehen. Die Höhe der Zusammenhänge der unmittelbaren

3 Erfahrungen mit der Sportaktivität nimmt dabei generell im Verlauf der Kursstunden zu und

4 erreicht substanzielle Varianzaufklärungen im Befinden zwischen 17\% und 27\% (zweite

5 Kurshälfte) bzw. zwischen 18\% und 29\% (Ende der Kursstunde). Im früheren

6 Stundenabschnitt klären die subjektiven Erfahrungskomponenten hingegen erst ca. 10\% der

7 Varianz im Befinden auf. Zu diesem Zeitpunkt bestimmt noch das Eingangs-Befinden die 8 affektive Valenz (28\%) und die Ruhe (33\%). Ebenso trägt die personale Voraussetzung des

9 Fitnesszustands im ersten Stundenabschnitt teilweise substanziell zur Varianzaufklärung des

10 Befindens bei (positive Aktivierung: 18\%, affektive Valenz: 8\%).

11 Für die verlaufsorientierte Betrachtung kann somit festgehalten werden, dass der Einfluss

12 der personalen Voraussetzungen im Befinden und im Fitnesszustand mit zunehmender Dauer

13 der Auseinandersetzung mit den Inhalten der Sportprogramme deutlich abnimmt und vom

14 psychophysischen Beanspruchungserleben während der Aktivität sowie sozial-kognitiven

15 Bewertungen der eigenen Kompetenz und des sozialen Umfelds in der Sportgruppe abgelöst

16 werden. Für die zukünftige verlaufsorientierte Forschung stellt sich die Herausforderung,

17 den Einfluss des Aktivitätstyps und seiner Inszenierung (z. B. gesellige Formen,

18 Spielformen, Aktivitäten eher mit Wettkampfbezug) systematisch mit einzubinden. In dieser

19 Studie wurden die Unterschiede zwischen den fünf Sportprogrammen lediglich als Störgröße

20 statistisch kontrolliert. Die Varianzaufklärungen durch die Art des Sportprogramms waren

21 hierbei zwar eher gering (affektive Valenz: 2-5\%; Ruhe: 0-4\%, positive Aktivierung: 5-9\%).

22 Durch eine systematischere Variation ließe sich aber der Einfluss des Aktivitätstyps in

23 Relation zu anderen Einflussfaktoren setzen. Hierbei wären auch andere personale

24 Voraussetzungen, wie individuelle Motive und Ziele, ins Kalkül zu ziehen, um sie etwa in

25 ihrer Passung zu den Stundeninhalten zu reflektieren (Sudeck \& Conzelmann, 2011).

26 Anwendung der Dual Mode Theory (Fragestellung 3)

27 Die Annahme konnte bestätigt werden, dass kognitive Bewertungen in Form der 28 wahrgenommenen Kompetenz einen größeren Zusammenhang mit dem Befinden bei 29 anstrengender im Vergleich zu moderater Belastungsintensität aufweisen. Dies bedeutet eine 30 erfolgreiche Anwendung der DMT im Kontext gruppenbasierter Freizeit- und 
1 Gesundheitsportprogramme. Die Belastungsintensität kann somit nicht nur als

2 eigenständiger (negativer) Einflussfaktor des Befindens betrachtet werden, sondern muss

3 auch als moderierender Faktor für die Beziehung zwischen kognitiven Bewertungen und

4 affektiven Reaktionen reflektiert werden. Für das positive Gruppenerleben zeigt sich ein

5 moderierender Einfluss der Belastungsintensität in eine andere Richtung: Das

6 Gruppenerleben ist vor allem dann für das Befinden relevant, wenn die Aktivität mit

7 moderater Intensität durchgeführt wird. Dies legt die Annahme nahe, dass bei anstrengender

8 Intensität die Bewertung von körper- und bewegungsassoziierten Merkmalen (wie die eigene

9 Kompetenz in der Bewegungsausführung) bedeutsamer ist und dies weniger Raum für das

10 positive Potenzial sozialer Interaktionen lässt.

11 Die Ergebnisse zur Bedeutung eines positiven Gruppenerlebens machen insgesamt

12 deutlich, dass der soziale Kontext im Freizeit- und Gesundheitssport eine nicht zu

13 vernachlässigende Größe ist, da zahlreiche präventive Bewegungsprogramme im

14 deutschsprachigen Raum gruppenbasiert realisiert werden (Pahmeier et al., 2012). In der

15 Erforschung affektiver Reaktionen in diesem Anwendungskontext müssen deshalb deutlich

16 stärker Forschungszugänge ergänzt werden, die den Einfluss des sozial-integrativen

17 Charakters gruppenbasierter Sportaktivitäten auf das Befinden adäquat berücksichtigen.

18 Die vorliegende Studie hat zwar einige der neueren Impulse in der Erforschung affektiver

19 Reaktionen umsetzen können. Die Studie weist aber insgesamt die Einschränkung auf, dass

20 keine physiologischen Verfahren zur Bestimmung der Belastungsintensität sowie zur

21 objektiven Bestimmung des Fitnesszustands eingesetzt wurden. Die objektive Bestimmung

22 der Intensität wird als ein Forschungsdesiderat im Kontext affektiver Reaktionen bei

23 Sportaktivitäten angesehen, um einheitliche Vorgehensweise in der Analyse von Aussagen

24 der DMT zu erreichen (Ekkekakis et al., 2011). Zukünftige Studien im Anwendungsbereich

25 des Freizeit- und Gesundheitssport sollten daher stärkeres Augenmerk auf eine physiologisch

26 basierte Kategorisierung der Intensität legen, um diesen Moderator valider bestimmen und

27 die Vermengung mit subjektiven Wahrnehmungen vermeiden zu können. Zudem ist auch für

28 Studiendesigns in Felduntersuchungen zu prüfen, inwieweit eine systematische Variation der

29 Intensität durch eine spezifische Belastungssteuerung herbeigeführt werden kann, um über

30 eine post-hoc-Gruppierung von Intensitätskategorien, wie in dieser Studie, hinauszugehen. 
1 Ferner bleibt insgesamt kritisch zu diskutieren, inwieweit eine Balance zwischen den

2 Anforderungen an die Kürze von mehrfachen Befragungen innerhalb von Sportprogrammen

3 in Felduntersuchungen einerseits und einer reliablen und validen Erfassung von Befindens-

4 und Erlebenskomponenten andererseits hergestellt werden kann (vgl. auch Fahrenberg,

5 2006). Die in dieser Studie eingesetzten kurzen (Befinden), modifizierten (Beanspruchung)

6 und teilweise modifizierten und noch nicht validierten Erhebungsvarianten

7 (wahrgenommener Fitnesszustand, Kompetenzerleben, Gruppenerleben) zeigten zwar gute

8 Verteilungseigenschaften und zufriedenstellende Reliabilitätskennwerte. Sie bedürfen jedoch

9 weiterer Validierungen im Anwendungskontext, wenngleich die vorgefundenen

10 substanziellen Zusammenhänge zwischen einzelnen Merkmalen sicherlich einen gewissen

11 Hinweis auf Konstruktvaliditäten enthalten.

12 Vor diesem Hintergrund hätte die vorliegende Studie in ihrem Design dahingehend

13 optimiert werden können, dass - trotz der Bedenken gegenüber der Belastung der Probanden

14 durch längere Befragungen während der Kursstunden - alle Erlebenskomponenten auch

15 während der Kursstunde erfasst worden wären. Dadurch wäre es möglich gewesen, für alle

16 Faktoren (hier auch das Kompetenzerleben und das positive Gruppenerleben) zeitlich

17 geordnete Sequenzen von Einflüssen auf affektive Reaktionen abbilden zu können.

Fazit

19 Für die Gestaltung von Programmen im Freizeit- und Gesundheitssport sensibilisieren die

20 Ergebnisse für die Erkenntnis, dass durchaus bekannte Einflussfaktoren des affektiven

21 Erlebens in Abhängigkeit der Intensität sowie im Verlauf einer Aktivität unterschiedlich zu

22 bewerten sind. Eine Beobachtung bzw. Befragung am Ende einer Kursstunde ist

23 möglicherweise bereits (positiv) verfälscht, wenn es um das Erleben der Aktivität selbst

24 geht. Bedeutsam ist deshalb, dass die relativ größte Variabilität bereits in frühen Abschnitten

25 der Kursstunde auftritt, wobei bei anstrengenden Belastungsintensitäten frühzeitig kognitive

26 Bewertungen der eigenen Kompetenz befindensrelevant werden. In Verbindung mit dem im

27 Mittel negativen Einfluss der Belastungsintensität werden dadurch Strategien bekräftigt, die

28 gerade bei (Wieder-)Einsteigern eine leichte bis moderate Intensität favorisieren, um

29 Überforderungssituationen vorzubeugen und die Ermöglichung von Erfolgserlebnissen zu

30 erleichtern (z. B. Brehm, 1998). Auf der anderen Seite verweist die DMT und ihre 
1 erfolgreiche Anwendung in dieser Studie darauf, dass die Belastungsintensität nicht per se

2 einen negativen Einfluss auf das Befinden hat, sondern dass anstrengende Aktivitäten

3 gepaart mit positiven kognitiven Bewertungen auch befindensförderlich sein können. Ferner

4 ist im Verlauf eines Freizeit- und Gesundheitssportprogramms die gezielte Beeinflussung

5 des affektiven Erlebens der Teilnehmenden immer wahrscheinlicher, da das Befinden

6 zunehmend von der Auseinandersetzung mit Inhalten und dem sozialem Kontext der

7 Aktivität abhängt und immer weniger von personalen Voraussetzungen im Befinden und

8 Fitnesszustand der Teilnehmenden.

9 Inwieweit die interindividuellen Unterschiede in affektiven Reaktionen systematisch

10 beeinflusst werden können, wird weiter Gegenstand der Forschung unter Berücksichtigung

11 der neueren konzeptionell-methodischen Impulse sein. Eine Verbindung mit der

12 Gesundheitsverhaltensforschung, die den sogenannten Exercise-Affect-Adherence-Link

13 (Williams, 2008) fokussiert, wird weiter konkretisieren können, inwieweit eine

14 Beeinflussung affektiver Reaktionen während und/oder nach sportlicher Betätigung für die

15 Bindung an regelmäßige Sportaktivitäten genutzt werden kann.

\section{Literatur}

17 Abele-Brehm, A. \& Brehm, W. (1986). Zur Konzeptualisierung und Messung von

18 Befindlichkeit - Die Entwicklung der „Befindlichkeitsskalen“(BFS). Diagnostica, 32, $19209-228$.

American College of Sports Medicine (ACSM) (2011). Quantity and quality of exercise for developing and maintaining cardiorespiratory, musculoskeletal, and neuromotor fitness in apparently healthy adults: Guidance for prescribing exercise. Medicine \& Science in Sport \& Exercise, 43,1334-1359.

Backhouse, S. H., Ekkekakis, P., Biddle, S. J. H., Foskett, A., \& Williams, C. (2007).

Bixby, W. R. \& Lochbaum, M. R. (2007). Affect responses to acute bouts of aerobic exercise in fit and unfit participants. An examination of opponent-process theory.

30 Borg, G. A. (1982). Psychophysical bases of perceived exertion. Medicine and Science in Sports and Exercise, 14 (5), 377-381. 
1 Brehm, W. (1998). Stimmung und Stimmungsmanagement. In K. Bös \& W. Brehm (Hrsg.), Gesundheitssport: Ein Handbuch (S. 201-211). Schorndorf: Hofmann.

3 Brehm, W., Wagner, P., Sygusch, R., Schönung, A. \& Hahn, U. (2005). Health promotion by means of health sport--a framework and a controlled intervention study with sedentary adults. Scand J Med Sci Sports, 15, 13-20.

Bryan, A., Magnan, R. E., Nilsson, R., Marcus, B. H., Tompkins, S. A., \& Hutchinson, K. E. (2011). The big picture of individual differences in physical activity change: A transdisciplinary approach. Psychology of Sport and Exercise, 12, 20-26.

Buskies, W. \& Boeckh-Behrens, W.-U. (2000). Gesundheitsorientiertes Fitnesstraining: Ausdauertraining, Ernährung und Entspannung. Lüneburg: Wehdemeiner \& Pusch.

Ekkekakis, P. (2003). Pleasure and displeasure from the body: Perspectives from exercise. Cognition and Emotion, 17, 213-239.

Ekkekakis, P. (2008). Affect circumplex redux: The discussion on its utility as a measurement framework in exercise psychology continues. International Review of Sport and Exercise Psychology, 31, 505-553.

Ekkekakis, P. \& Petruzello, S. J. (1999). Acute aerobic exercise and affect: current status, problems and prospects regarding dose-response. Sports Medicine, 28, 337-374.

Ekkekakis, P. \& Petruzello, S. J. (2000). Analysis of the affect measurement conundrum in exercise psychology: I. Fundamental issues. Psychology of Sport and Exercise, 1, 7188.

Ekkekakis, P., Parfitt, G. \& Petruzzello, S. J. (2011). The pleasure and displeasure people feel when the exercise at different intensities. Sports Medicine, 41 (8), 641-671.

Fahrenberg, J. (2006). Einstufung der Befindlichkeit - Einzelne Items oder Skalen wie ADACL und PANAS? [Elektronische Version]. Zugriff unter http://www.ambulatoryassessment.org/typo3/ambulatory/fileadmin/user_upload/statements/items_oder_skalen_

30 Jackson, S. A., \& Marsh, H. (1996). Development and validation of a scale to measure optimal experience: The Flow State Scale. Journal of Sport \& Exercise Psychology, 18, $17-35$. 
1 Kircaldy, B. D. \& Shephard R. J. (1990). Therapeutic implications of exercise. International Journal of Sport Psychology, 21, 165-184.

3 Kleinert, J. (2006). Adjektivliste zur Erfassung der Wahrgenommenen Körperlichen Verfassung (WKV): Skalenkonstruktion und erste psychometrische Befunde. Zeitschrift für Sportpsychologie, 13, 156-164.

Kwan, B. M., \& Bryan, A. D. (2010). Affective response to exercise as a component of exercise motivation: Attitudes, norms, self-efficacy, and temporal stability of intentions. Psychology of Sport and Exercise, 11, 71-79.

Lehnert, K., Sudeck, G. \& Conzelmann, A. (2012). Subjective well-being and exercise in the

Mäder, U., Martin, B., Schutz, Y., \& Marti, B. (2006). Validity of four physical activity questionnaires in middle-aged persons. Medicine \& Science in Sports \& Exercise, 37, 1255-1266.

McAuley, E., Blissmer, B., Katula, J. \& Duncan, T. E (2000). Exercise environment, selfefficacy, and affective response to acute exercise in older adults. Psychology and Health, 15, 341-355.

Morgan, W. P. \& O’Connoe, P. J. (1988). Exercise and mental health. In R. K. Dishman (Ed.), Exercise adherence: its impact on public health. Champaign (IL): Human Kinetics.

Netz, Y., Wu, M., Becker, B. \& Tenenbaum, G. (2005). Physical activity and psychological well-being in advanced age: A meta-analysis of intervention studies. Psychology and Aging, 20, 272-284.

Rheinberg, F. (2004). Motivationsdiagnostik. Göttingen: Hogrefe.

Rose, E. A. \& Parfitt, G. (2007). A quantitative analysis and qualitative explanation of the intensities. Journal of Sport \& Exercise Psychology, 29 (3), 281-309. 
1 Rose, E.A. \& Parfitt, G. (2012). Exercise experience influences affective and motivational outcomes of prescribed and self-selected intensity exercise. Scandinavian Journal of Medicine \& Science in Sports, 22, 265-277.

4 Schimmack, U., \& Grob, A. (2000). Dimensional models of core affect: A quantitative comparison by means of structural equation modeling. European Journal of Personality, 14, 325-345.

Schlicht, W. \& Reicherz, A. (2012). Sportliche Aktivität und affektive Reaktionen. In R. Fuchs \& W. Schlicht (Hrsg.). Seelische Gesundheit und sportliche Aktivität (S. 12-33). Göttingen: Hogrefe.

Schlicht, W., Ebner-Priemer, U. W. \& Kanning, M. (2013). Ecological momentary assessment and intervention in physical activity and well-being: affective reactions, social-cognitive factors, and behaviors as determinants of physical activity and exercise. Frontiers in Psychology, 4, 916. doi: 10.3389/fpsyg.2013.00916

Sudeck, G., Lehnert, K. \& Conzelmann, A. (2011). Motivbasierte Sporttypen - Auf dem

Sudeck, G. \& Conzelmann, A. (2011). Motivbasierte Passung von Sportprogrammen:

5
Explizite Ziele und Motive als Moderator von Befindlichkeitsveränderungen durch sportliche Aktivität. Sportwissenschaft, 41, 175-189. Weg zur Person-orientierung im zielgruppenspezifischen Freizeit- und Gesundheitssport. Zeitschrift für Sportpsychologie, 18 (1), 1-17.

Van Landuyt, L. M., Ekkekakis, P., Hall, E. E. \& Petruzzello, S. J. (2000). Throwing the mountains into the lakes: On the perils of nomothetic conceptions of the exerciseaffect relationship. Journal of Sport and Exercise Psychology, 22, 208-234.

Welch, A. S., Hulley, A. \& Beauchamp, M. (2010). Affect and self-efficacy responses during moderate-intensity exercise among low-active women: The effect of cognitive appraisal. Journal of Sport and Exercise Psychology, 32, 154-175

Wilhelm, P., \& Schoebi, D. (2007). Assessing mood in daily life. Structural validity, sensitivity of change, and reliability of a short-scale to measure three basic dimensions of mood. European Journal of Psychological Assessment, 23, 258-267.

Williams, D. M. (2008). Exercise, affect, and adherence: An integrated model and a case for self-paced exercise. Journal of Sport and Exercise Psychology, 30, 471-496. 
1 Williams, D. M., Dunsiger, S., Jennings, E. \& Marcus, B. (2012). Does affective valence 2 during and immediately following a 10-Min walk predict concurrent and future 3 physical activity? Annals of Behavioral Medicine, 44, 43-51. 
1 Abbildung 1. Übersicht über Studienablauf und Teilnehmende sowie Vollständigkeit der Datenerhebungen innerhalb der Sporteinheiten

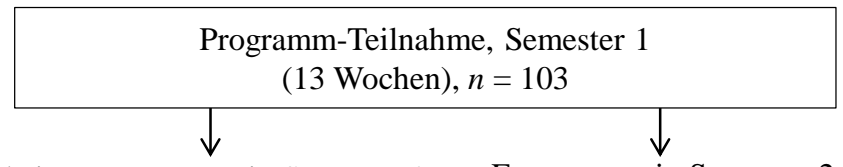

keine Fortsetzung in Semester 2 Fortsetzung in Semester $2 \quad$ Neueinstieg

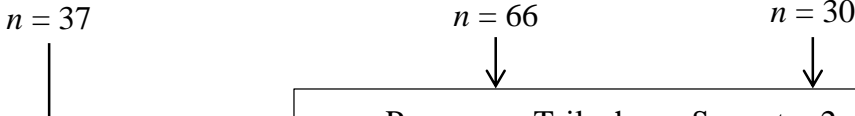

Programm-Teilnahme, Semester 2 (14 Wochen), $n=95$

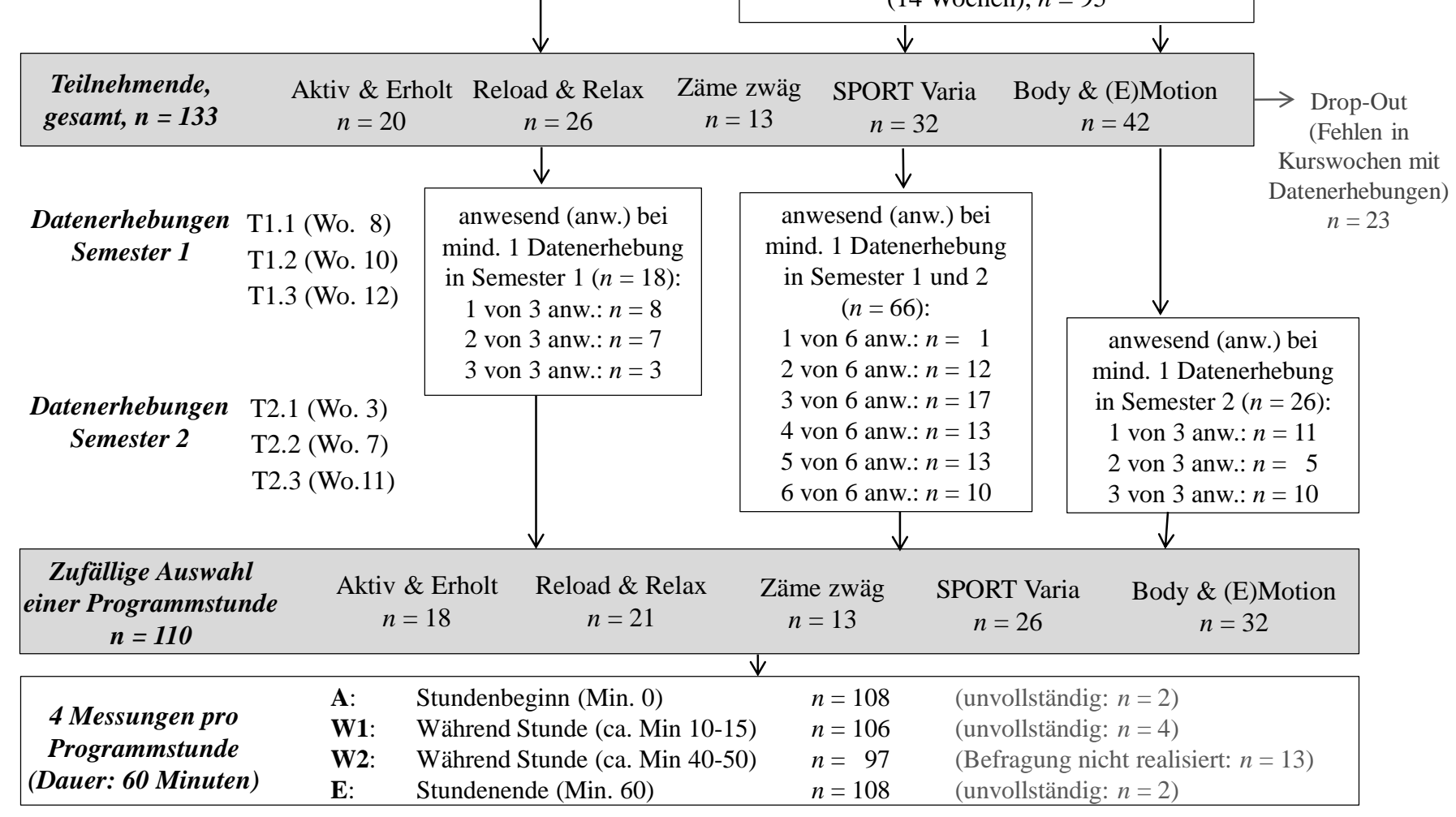


1 Abbildung 2. Befindenswerte im Verlauf der Kursstunde (mit statistischer Kontrolle der

2 Eingangswerte und der Kursart; W1 = Während-Messung 1, W2 = Während-Messung 2; E =

3 Ende Kursstunde)
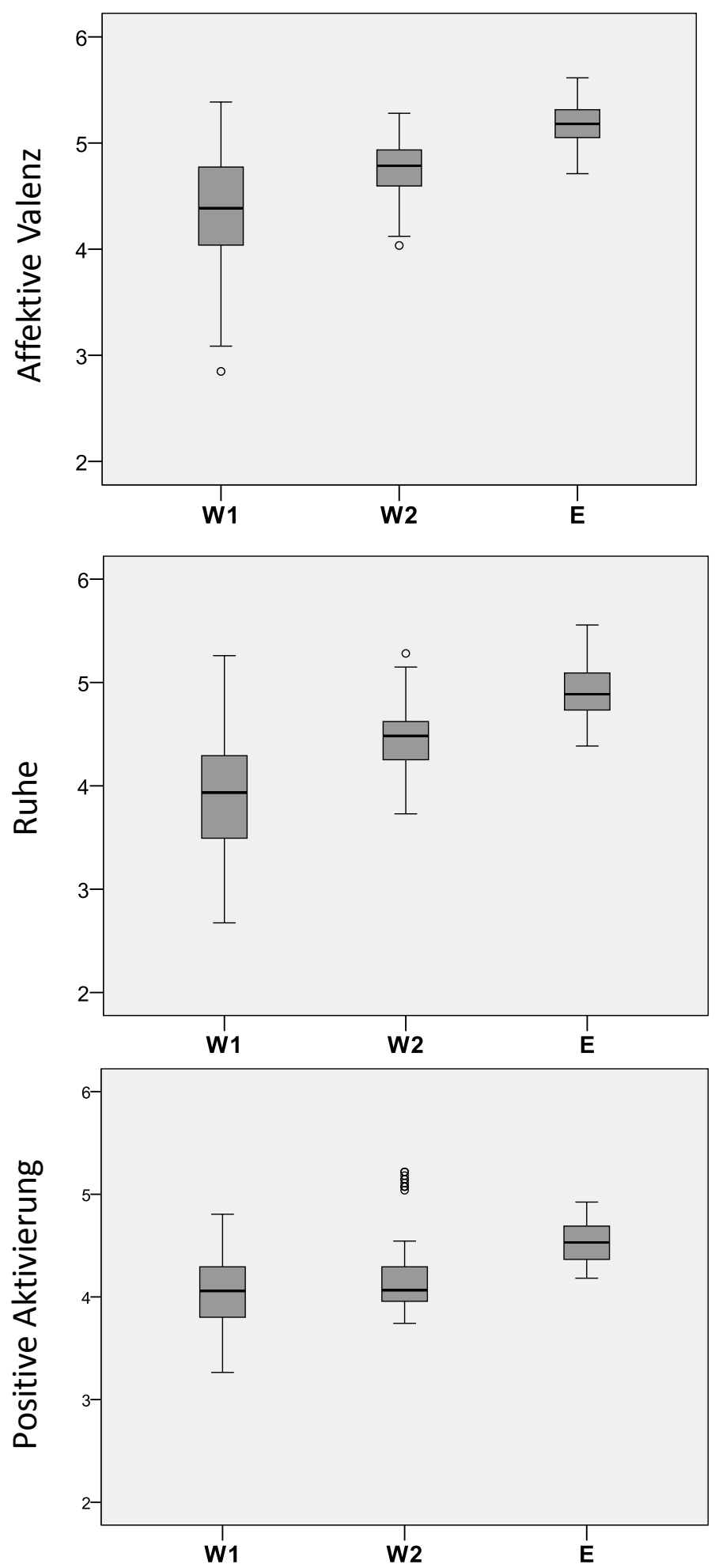
Tabelle 1. Übersicht über die Zeitpunkte der Erfassung der Untersuchungsmerkmale am Anfang (A), während (W1, W2) und am Ende der Sportaktivität (E) sowie deskriptive Kennwerte und interne Konsistenz der Erhebungsverfahren

\begin{tabular}{|c|c|c|c|c|c|c|c|c|c|c|}
\hline Merkmal & $\begin{array}{l}\text { Anzahl } \\
\text { Items }\end{array}$ & Zeitpunkt & $n$ & $\begin{array}{c}\text { Interne } \\
\text { Konsistenz }\end{array}$ & $M$ & $S D$ & Min & Max & Schiefe & Exzess \\
\hline \multirow[t]{4}{*}{ Affektive Valenz } & 2 & $\mathrm{~A}$ & 108 & $r=.79$ & 4.07 & 1.21 & 0.5 & 6 & -0.42 & 0.02 \\
\hline & & W1 & 106 & $r=.69$ & 4.39 & 1.08 & 1.5 & 6 & -0.52 & -0.21 \\
\hline & & W2 & 97 & $r=.56$ & 4.75 & 0.78 & 2 & 6 & -0.82 & 1.23 \\
\hline & & $\mathrm{E}$ & 108 & $r=.75$ & 5.18 & 0.73 & 3 & 6 & -0.83 & 0.54 \\
\hline \multirow[t]{4}{*}{ Ruhe } & 2 & $\mathrm{~A}$ & 108 & $r=.66$ & 3.63 & 1.26 & 0.5 & 6 & -0.15 & -0.49 \\
\hline & & W1 & 106 & $r=.50$ & 3.92 & 0.92 & 2 & 6 & 0.17 & -0.31 \\
\hline & & W2 & 97 & $r=.66$ & 4.44 & 0.76 & 2.5 & 6 & -0.31 & -0.52 \\
\hline & & $\mathrm{E}$ & 108 & $r=.70$ & 4.90 & 0.82 & 2.5 & 6 & -0.62 & 0.02 \\
\hline \multirow[t]{4}{*}{ Positive Aktivierung } & 2 & A & 108 & $r=.68$ & 3.45 & 1.20 & 0.5 & 6 & -0.30 & -0.43 \\
\hline & & W1 & 106 & $r=.62$ & 4.04 & 0.98 & 0.5 & 6 & -0.72 & 1.03 \\
\hline & & W2 & 97 & $r=.75$ & 4.19 & 1.16 & 0 & 6 & -0.96 & 1.56 \\
\hline & & $\mathrm{E}$ & 108 & $r=.62$ & 4.53 & 1.03 & 1 & 6 & -0.98 & 0.99 \\
\hline wahrgenommener Fitnesszustand & 3 & $\mathrm{~A}$ & 108 & $\alpha=.89$ & 2.56 & 0.92 & 0.33 & 4.67 & -0.10 & -0.25 \\
\hline \multirow[t]{3}{*}{ subjektive Beanspruchung } & 1 & W1 & 106 & $\begin{array}{l}-- \\
\end{array}$ & 3.57 & 1.23 & 1 & 6 & -0.10 & -0.41 \\
\hline & & W2 & 97 & --- & 4.15 & 1.18 & 1 & 7 & -0.15 & 0.17 \\
\hline & & $\mathrm{E}$ & 108 & --- & 3.15 & 1.33 & 1 & 7 & 0.16 & -0.37 \\
\hline wahrgenommene Kompetenz & 1 & $\mathrm{E}$ & 108 & --- & 3.98 & 0.85 & 1 & 5 & -0.79 & 0.75 \\
\hline positives Gruppenerleben & 2 & $\mathrm{E}$ & 108 & $r=.50$ & 4.06 & 0.85 & 1.5 & 5 & -1.21 & 1.31 \\
\hline
\end{tabular}


Tabelle 2. Blockweise lineare Regressionen zur Bestimmung von Einflussfaktoren auf die Befindensdimensionen affektive Valenz, Ruhe und positive Aktivierung im Verlauf der Kursprogramme (mit Einbezug der Kovariate Kursprogramm, Step 1)

\begin{tabular}{|c|c|c|c|c|c|c|}
\hline \multirow[b]{3}{*}{ Prädiktoren } & \multicolumn{6}{|c|}{ AV: Affektive Valenz } \\
\hline & \multicolumn{2}{|c|}{ W1 } & \multicolumn{2}{|c|}{$\mathrm{W} 2$} & \multicolumn{2}{|c|}{ E } \\
\hline & $\Delta R^{2}$ & $\beta$ & $\Delta R^{2}$ & $\beta$ & $\Delta R^{2}$ & $\beta$ \\
\hline Step 2 & $.28 *$ & & $.07 *$ & & $.04 *$ & .16 \\
\hline $\begin{array}{l}\text { Eingangswert } \\
\text { affektive Valenz }\end{array}$ & & $.47 *$ & & $.23^{*}$ & & \\
\hline Step 3 & $.08 *$ & & .03 & & .02 & \\
\hline wahrg. Fitnesszustand & & $.22 *$ & & .10 & & .04 \\
\hline Step 4 & $.11 *$ & & $.27 *$ & & $.29 *$ & \\
\hline Beanspruchung & & .01 & & $-.20 *$ & & -.10 \\
\hline Kompetenzerleben & & $.25^{*}$ & & $.26^{*}$ & & $.24^{*}$ \\
\hline positives Gruppenerleben & & $.20 *$ & & $.36^{*}$ & & $.45^{*}$ \\
\hline \multirow[t]{3}{*}{ Total $R^{2}$} & $.49 *$ & & $.42 *$ & & $.37 *$ & \\
\hline & \multicolumn{6}{|c|}{ AV: Ruhe } \\
\hline & \multicolumn{2}{|c|}{ W1 } & \multicolumn{2}{|c|}{ W2 } & \multicolumn{2}{|c|}{ E } \\
\hline Prädiktoren & $\Delta R^{2}$ & $\beta$ & $\Delta R^{2}$ & $\beta$ & $\Delta R^{2}$ & $\beta$ \\
\hline Step 2 & $.33 *$ & & $.17 *$ & & $.05^{*}$ & \\
\hline Eingangswert Ruhe & & $.61 *$ & & $.49 *$ & & $.29 *$ \\
\hline Step 3 & .02 & & .00 & & .00 & \\
\hline wahrg. Fitnesszustand & & .06 & & -.02 & & -.16 \\
\hline Step 4 & $.08 *$ & & $.17^{*}$ & & $.18^{*}$ & \\
\hline Beanspruchung & & $-.18 *$ & & -.16 & & -.11 \\
\hline Kompetenzerleben & & .16 & & $.31 *$ & & $.27 *$ \\
\hline positives Gruppenerleben & & .12 & & .18 & & $.28 *$ \\
\hline \multirow[t]{3}{*}{ Total $R^{2}$} & $.46^{*}$ & & $.33 *$ & & $.27 *$ & \\
\hline & \multicolumn{6}{|c|}{ AV: Positive Aktivierung } \\
\hline & \multicolumn{2}{|c|}{ W1 } & \multicolumn{2}{|c|}{ W2 } & \multicolumn{2}{|c|}{ E } \\
\hline Prädiktoren & $\Delta R^{2}$ & $\beta$ & $\Delta R^{2}$ & $\beta$ & $\Delta R^{2}$ & $\beta$ \\
\hline Step 2 & $.08 *$ & & .01 & & .00 & \\
\hline $\begin{array}{l}\text { Eingangswert } \\
\text { positive Aktivierung }\end{array}$ & & .10 & & $-.19 *$ & & .07 \\
\hline Step 3 & $.18 *$ & & $.10 *$ & & $.04 *$ & \\
\hline wahrg. Fitnesszustand & & $.42 *$ & & $.26^{*}$ & & .14 \\
\hline Step 4 & $.09 *$ & & $.23 *$ & & $.19 *$ & \\
\hline Beanspruchung & & -.13 & & $-.35^{*}$ & & .00 \\
\hline Kompetenzerleben & & .16 & & $.25^{*}$ & & $.29 *$ \\
\hline positives Gruppenerleben & & $.18^{*}$ & & $.17^{*}$ & & $.30 *$ \\
\hline Total $R^{2}$ & $.40 *$ & & $.43 *$ & & $.28 *$ & \\
\hline
\end{tabular}

Anmerkung. Es werden die Regressionsgewichte berichtet, die sich als Resultat des vierten Schritts ergaben; $* p<.05$ 
Tabelle 3. Blockweise lineare Regressionsanalysen zur Bestimmung von Einflussfaktoren der affektiven Valenz im Verlauf der Sportaktivität in Abhängigkeit der Beanspruchung (mit Einbezug der Kovariaten Kursprogramm; Step 1)

\begin{tabular}{|c|c|c|c|c|c|c|c|c|}
\hline \multirow[b]{3}{*}{ Prädiktoren } & \multicolumn{4}{|c|}{ W1 } & \multicolumn{4}{|c|}{ W2 } \\
\hline & \multicolumn{2}{|c|}{$\begin{array}{l}\text { moderat } \\
(n=62)\end{array}$} & \multicolumn{2}{|c|}{$\begin{array}{l}\text { anstrengend } \\
\quad(n=21)\end{array}$} & \multicolumn{2}{|c|}{$\begin{array}{l}\text { moderat } \\
(n=52)\end{array}$} & \multicolumn{2}{|c|}{$\begin{array}{l}\text { anstrengend } \\
\qquad(n=33)\end{array}$} \\
\hline & $\Delta R^{2}$ & $\beta$ & $\Delta R^{2}$ & $\beta$ & $\Delta R^{2}$ & $\beta$ & $\Delta R^{2}$ & $\beta$ \\
\hline Step 2 & $.43^{*}$ & & .06 & & $.09 *$ & & .01 & \\
\hline $\begin{array}{l}\text { Eingangswert } \\
\text { affektive Valenz }\end{array}$ & & $.68^{*}$ & & .33 & & .19 & & .21 \\
\hline Step 3 & .01 & & .16 & & .03 & & .00 & \\
\hline wahrg. Fitnesszustand & & .02 & & .23 & & .20 & & .00 \\
\hline Step 4 & .05 & & $.32 *$ & & $.28 *$ & & $.21 *$ & \\
\hline Kompetenzerleben & & .10 & & $.54 *$ & & .14 & & $.45^{*}$ \\
\hline positives Gruppenerleben & & .19 & & .22 & & $.50 *$ & & .15 \\
\hline Total $R^{2}$ & $.50^{*}$ & & $.70^{*}$ & & $.51^{*}$ & & .37 & \\
\hline
\end{tabular}

Anmerkung. Es werden die Regressionsgewichte berichtet, die sich als Resultat des vierten Schritts ergaben;

$$
* p<.05
$$

